\title{
DID.VALUE: AN INTERNATIONAL PROJECT FOCUSED ON BUILDING AN OPEN DIGITAL FRAMEWORK FOR EDUCATIONAL RESOURCES ON INCLUSIVE JOBS AND TRAINEESHIPS FOR YOUNG PEOPLE WITH DISABILITIES
}

\author{
Maria Potes Barbas', Pedro Matos ${ }^{1}$ \\ ${ }^{1}$ Instituto Politécnico de Santarém - Escola Superior de Educação (PORTUGAL)
}

\begin{abstract}
The study in question focuses on the development of a digital and open framework, with the objective of being an educational resource for young adults over the age of 18 years and with intellectual disabilities. This project is part of an international initiative called DID. Value and aims to empower young adults for inclusive internship and employment scenarios. This framework consists of an adapted model of digital training, in which the adopted methodology goes through a phased process, step-by-step and using the various digital tools, to make the learning experience the most intuitive possible. Furthermore, this model used two resources widely utilised in online education, Perusall and Moodle to establish a platform rich in interactive content. In addition, other features such as H5P, interactive manuals, videos and virtual scenarios were used to enable a unique experience for participants, including real-life contexts, with challenges, activities and exercises that would lead participants to have an active and contributory position. Thus, we will analyse the building model of this framework, understand the best practices achieved in terms of content and structure for online teaching and training. In addition, we will make a brief analysis of the use of some specific digital resources and why they are best suited for this type of target audience.
\end{abstract}

Keywords: Digital Framework, E-learning Contents, E-learning Frameworks, Digital Learning, Students with Disabilities

\section{INTRODUCTION}

As the digital society expands, as do all of its players, it is important to accompany this growth with the appropriate means and resources. In the case of this study and taking into account the target audience (young adults with disabilities), the issue to be explored is the creation of an open digital framework that integrates intuitive educational content from a labour market perspective.

As such, the use of digital learning platforms has become increasingly common as an educational strategy. In addition to enabling more analytical and interactive monitoring by trainers, it also allows the use of numerous digital resources that facilitate the learning process. Still, the importance of these digital-based learning models has applied a sense of development more suited to the needs of its target audience. Not in the least because, the design of teaching activity and the flexible application of technology tools or digital learning therefore become the primary issues for current information technology integrated education [1].

It is also added that the implementation of this type of digital frameworks broadens the teachinglearning aspect of the students. In this case, and since we are referring in particular to young adults with disabilities, the possibility of including in a curriculum features such as: innovative content design, delivery adapted systems and other intuitive and accessible components, can extend the walls of the classroom to areas beyond, in which students transfer and connect course content with the professional world [2].

Another aspect to take into account in this type of study is the customisation factor. Being that personalized learning models can give each student differentiated learning experiences based on their needs, interests, and strengths - including students with disabilities [3]. That said, the importance of ensuring a multi-level, yet holistic approach allows for a model in which it includes learner profiles capture skills, gaps, strengths, weaknesses, interests, and aspirations of each student, and personal learning paths match diverse learning experiences to individual needs, goals, and objectives [3]. Based on these characteristics another positive aspect of including a consistent and adapted digital learning framework is that learning becomes much less restrictive, and this is ideal for learners with disabilities. 


\section{STUDY METHODOLOGICAL PROCESS}

The methodology present in this study assumed a dynamic and evolutionary aspect. Since this is a digital project, it is important to understand that it cannot become stagnant. As new milestones and performance indicators are established, it is necessary to design a monitoring strategy appropriate to the different outcomes of the project. As such, a methodological process was established based on an approach that prioritises organizational key performance indicators (KPIs) in terms of the criteria of SMART (Specific, Measurable, Attainable, Realistic, Time-sensitive) goal setting [4].

Thus, obtaining an overview of the study we have as criteria (KPIs) the following points:

- Specific - Development of a digital and open framework for edutainment resources in employment and internships.

- Measurable - Creation of digital content (practical guides, tutorials, and videos) that could be incorporated into the Platform as didactic/pedagogical resources.

- Attainable - Implementation of a training programme adapted to the participants' needs (young adults with disabilities), including employment and internships.

- Realistic - Implementation of a pilot experience as a test to the prototype of the digital framework developed and respective learning contents.

- Time-sensitive - Adaptation of all these milestones to the timeframe foreseen in the project for the development of the various sprints in terms of inputs.

Having achieved this methodological process, which served as a structural basis for the study and respective project, it was verified throughout the inputs and performance indicators achieved the need to include strategies that would allow a more adequate learning flow for the target audience. Thus, it was started by establishing one of the main objects of the study that would interact directly with the trainees, that is, a Digital Delivery System. This system involves the use of a content management or learning management utilities that display, provide access to, or otherwise render digital materials for students' use. Most of these systems require an individual student logon via username/password or unique student identification number, and record and display student usage and achievement data [5].

For the purposes of this study and respective project, the Digital Delivery System used was Moodle because, after a more careful analysis of the needs of the target audience, it was considered the best tool for the construction of the open digital framework. Besides, the Moodle platform is one of the most capable when it comes to applying the Universal Instructional Design Principles because: i) it promotes an equitable use; ii) it allows a flexible use; iii) it is simple and intuitive for the vast majority of students (with or without disabilities); iv) it allows the information to be perceptible and accessible through accessibility integrator modules; $v$ ) it is error tolerant and guides users towards its respective solution; vi) it involves a very low physical and technical effort; vii) it includes a practical, dynamic and intuitive instructional design climate [6].

Still on the methodological process adopted in this study, it is important to mention another aspect that enabled its development. In this case, we refer to the Competency/Proficiency-Based Learning which in this curricular structure would allow monitoring students' progress based on daily progress towards their personal, technical, and pedagogical goals. Other important aspect is that in this framework there were not designated proficiency levels - not by grades - but by the trainee's movement through the course being based on their own evidence and paced learning [5]. This method in specific allowed a more personal learning bubble, respecting the students view on how they would acquire, produce, or even apply their own skills or knowledge through the different learning scenarios.

Finally, the methodological process also included a method that would allow the different actors to have a common base, both in terms of learning and facing the challenges of incorporating a totally mediated Teaching through online. Thus, the model Universal Design for Learning (UDL), allowed the implementation of a scientifically based framework focused on supporting the variability of every learner through proactive and iterative design that integrates multiple means of engagement, representation of information, and action and expression of understanding [5], being an adequate solution for the step-by-step learning method of students with disabilities. 


\section{INITIAL FINDINGS AND RESULTS}

The results of this study are still mostly inconclusive as it is still in a pilot phase. However, some initial considerations and results emerge which are important to be analysed and discussed. Regarding the dimension of the teaching-learning process, on the employment component and the inclusive traineeships, the digital open framework includes a set of learning modules, based on a step-by-step methodology, with the objective of accompanying the trainees in the resolution of their online activities.

In this way, the modules have been divided into different topics keeping in mind a diverse and adequate offer to the different needs of the trainees. The topics in question are:

a) Social, pedagogical, and digital Literacy Skills - focusing on the acquisition of social, pedagogical skills and basic digital concepts.

b) Working relationships, teamwork or collaborative and community building and networking - focusing on developing skills related to collaboration, networking, peer work and brainstorming.

c) Creative skills, emotional intelligence, and cultural awareness - focusing on the creative part of the trainees and the development of skills related to emotional management and collective cultural awareness.

It is worth noting that all these modules were based on established European frameworks such as EntreComp, LifeComp and DigiComp in order to enrich the learning content produced and to address some of the priorities at European level. Furthermore, all modules followed a flexible structure that included (i) an introductory video, (ii) several interactive infographics, (iii) a pedagogical guide, (iv) a library of digital resources and (v) a digital assessment. This digital assessment was responsible for the intermediate evaluation of the trainees since it integrated in the platform a brief quiz and a module evaluation form. Thus, allowing trainers to understand and monitor the evolution of the learning curve of the various trainees.

Regarding the technological component and the main results identified, we can classify them, taking into account the nature of the framework, as a considerably important basis for the production and presentation of content. This is because two digital tools were used: Perusall and H5P as a way to make the resources used (PDF, images, videos, among others) more intuitive for the trainees. The use of Perusall was particularly useful because it allowed a greater variety of interactions with contents where reading, writing and notes take the major part as a teaching-learning strategy. In fact, this tool is seen by the trainees as an agile, intuitive, and easily accessible way when the objective is to read, analyse and comment an online document [7]. Thus, we consider Perusall an essential tool for the strategy of content creation for learners with disabilities.

As for the use of the other digital tool, the H5P, it was found to be relevant to the extent that it allowed for the creation of automated, interactive content that would allow for a more enriching and active experience from the learner's point of view. In this sense, H5P became very useful for (i) the development of images in $360^{\circ}$ view, (ii) the implementation of hotspots in images, and (iii) content based on automatic tasks that provided feedback at the moment of execution by the trainee. Also, this tool was seen as a multimodal strategy because it allowed the integration of the basic learning model component and, at the same time, the full connection with interactive multimedia content [8] resulting in a more immersive environment for the learner.

In short, another visible technological component in the production of this digital framework was the incorporation with interactive content packages in SCORM format. This practice allowed for a speeding up of the process of importing teaching resources into the framework.

\subsection{Focus group main findings}

Also, for the purpose of results and final considerations, it was found to be important to apply an evaluation questionnaire on the digital framework. To this end, a focus group was defined, in this case 6 students with disabilities (4 with Down syndrome and 2 with autism), in order to evaluate the framework based on four main criteria: (i) Accessibility, (ii) Readability, (iii) Functionality and (iv) Intuitiveness. In this sense, students would have to assess each dimension using a numerical scale from 1 to 5 . With 1 representing Very Little and 5 representing Very Much. This analysis became considerably important in order to understand the shortcomings noted by users and how solutions could be applied to remedy them. 
Table 1. Digital Framework Evaluation Results

\begin{tabular}{l|c|c|c|c}
\hline \hline & Accessibility (1-5) & Legibility (1-5) & Functionality (1-5) & Intuitiveness (1-5) \\
\hline Student 1 (DS) & 3 & 5 & 4 & 4 \\
\hline Student 2 (DS) & 3 & 5 & 3 & 4 \\
\hline Student 3 (Autism) & 3 & 4 & 3 & 3 \\
\hline Student 4 (DS) & 3 & 4 & 4 & 3 \\
\hline Student 5 (Autism) & 4 & 3 & 4 & 4 \\
\hline Student 6 (DS) & 3 & 3 & 4 & 3 \\
\hline \hline
\end{tabular}

After analysing the data, we found that:

a) The Legibility and Functionality criteria were the ones that presented the highest score in terms of simple weighted average. Respectively Legibility $\bar{X}=4$ and Functionality $\bar{X}=3.7$.

b) The Accessibility and Intuitiveness criteria were the ones that presented the lowest score in terms of simple weighted average. Respectively Accessibility $\bar{X}=3.2$ and Intuitiveness $\bar{X}=3.5$.

c) Students 1 and 2 considered the Legibility criterion to be the most verifiable in the framework.

d) Student 3 equally considered the Legibility criterion as the most suitable in the framework.

e) Student 4 considered the criteria Legibility and Functionality as the most adequate in the framework.

f) Student 5 presented another trend, evaluating the criteria of Accessibility, Functionality, and Intuitiveness with the best score.

g) Student 6 indicated only Functionality as the most adequate criteria in the framework.

Thus, we can see that the Accessibility criterion is still the one that needs a greater margin of progress. In this way, other types of strategies or more appropriate solutions will be necessary to integrate into the digital framework. Since the options of contrast variation, colour difference, increased text size and other aspects are not yet necessary for these students to consider complete accessibility.

\section{CONCLUSIONS}

With this initial study we can conclude that developing a digital framework that includes the inclusive teaching-learning component is still a challenge that needs further solutions. This is because inclusive education encompasses a number of essential aspects, such as: recognising, accommodating and meeting the learning needs of all students. It means acknowledging that students with disabilities have a range of individual learning needs and that they are members of diverse communities [9]. Thus, this raises several issues. One of the most fundamental is the support that the e-trainer has to provide to learners with disabilities. Although online training is enriching from several points of view, namely: (i) in the synchronous and asynchronous component; (ii) in the more personalized monitoring; (iii) in the dynamics of the construction and presentation of interactive content and (iv) even in the very involvement in a practical digital community, these are not without challenges for a target audience whose basic digital skills are still underdeveloped. This leads to the need for future studies of this nature to be more focused and to explore these topics further. In order to develop a more consistent and robust critical mass on the digital solutions to be applied for the creation of this type of frameworks based on training and learning for students with disabilities. However, we cannot fail to observe the high growth and popularity that this type of strategy has been assuming in training contexts for sensitive target audiences. In fact, these means, and resources increase flexibility, efficiency, and foster engagement in learning [10]. It should also be noted that this study crossed only information from a very specific focus group, which may lead to a certain inconsistency, in terms of results, on the part of other working groups. Regardless of this situation, there is still a gap in the field of accessibility and the respective components inherent to this practice. These need to be properly identified and 
addressed in order to create more adequate solutions to the different learning needs of students with disabilities.

\section{REFERENCES [ARIAL, 12-POINT, BOLD, LEFT ALIGNMENT]}

[1] M.H. Lin, H.C. Chen, K.S. Liu, "A Study of the Effects of Digital Learning on Learning Motivation and Learning Outcome," EURASIA Journal of Mathematics Science and Technology Education, vol. 13, no. 7, pp. 3553-3564, 2017.

[2] J.M. Balzotti, L.B. McCool, " Using Digital Learning Platforms to Extend the Flipped Classroom," Business and Professional Communication Quarterly, vol. 79, no. 1, pp.68-80, 2016.

[3] M. Worthen, "The future of personalized learning for students with disabilities,". State Education Standard, vol. 16, no.3, p.35, 2016

[4] A. Shahin, M.A. Mahbod, "Prioritization of key performance indicators: An integration of analytical hierarchy process and goal setting," International Journal of Productivity and Performance Management, vol. 56, no.3, pp. 226-240, 2007.

[5] J.D. Basham, W. Stahl, K.R. Ortiz, M.F. Rice, S.J. Smith, "Equity matters: Digital and online learning for students with disabilities". The Center on Online Learning and Students with Disabilities, 2015.

[6] T. Elias," Universal instructional design principles for Moodle", The International Review of Research in Open and Distributed Learning, vol. 11, no. 2, pp. 110-124, 2010.

[7] A.J. Clarke, "Perusall: Social learning platform for reading and annotating", Journal of Political Science Education, vol. 17, no. 1, pp. 149-154, 2021.

[8] L.N. Amali, N.T. Kadir, M. Latief, "Development of e-learning content with H5P and iSpring features”, Journal of Physics: Conference Series, vol. 1387, no. 1, pp. 12-19, 2019.

[9] D. Bjekić, S. Obradović, M. Vučetić, M. Bojović, "E-teacher in Inclusive e-education for Students with Specific Learning Disabilities", Procedia - Social and Behavioral Sciences, vol. 128, pp. 128-133, 2014.

[10] A.I. Hashey, S. Stahl, "Making Online Learning Accessible for Students With Disabilities", TEACHING Exceptional Children, vol. 46, no. 5, pp. 70-78, 2014. 